\title{
RDUS
}

Revue de DROIT

UNIVERSITÉ DE SHERBROOKE

Titre : $\quad$ LA CHARTE CONSTITUTIONNELLE DE 1982 ET LA DÉMOCRATIE ÉLECTORALE CANADIENNE

Auteur(s): $\quad$ Patrice GARANT

Revue : $\quad$ RDUS, 1990-1991, volume 21, numéro 2

Pages : $\quad 429-469$

ISSN : $\quad 0317-9656$

Éditeur : $\quad$ Université de Sherbrooke. Faculté de droit.

URI : $\quad$ http://hdl.handle.net/11143/13443

DOI : https://doi.org/10.17118/11143/13443 
Page vide laissée intentionnellement. 


\section{LA CHARTE CONSTITUTIONNELLE DE 1982 ET LA DÉMOCRATIE ÉLECTORALE CANADIENNE}

par Patrice GARANT*

La Charte de 1982 constitutionnalise le droit de suffrage, le droit de candidature aux élections législatives, le droit à la liberté d'expression politique et le droit à l'égalité. Plusieurs dispositions de nos lois électorales ont jusqu'ici fait l'objet de contestations dont certaines ont porté sur des questions faciles, tels les titulaires du droit de vote, les conditions d'exercice du suffrage; d'autres questions sont plus complexes, tels les limites au droit de candidature, les congés électoraux et surtourt le plafonnement et le contrôle des dépenses électorales, le financement des candidats et partis.

The Charter has up to now raised a few important challenges to our electoral laws. The voting rights question has been settled easily enough, as much as the question of residence requirements, registration, proxy and special voting; the question of redistribution critera and redistribution process is surely more complex. Other sections have been challenged, namely the limitations of political freedom of public servants and the general access to political leaves without pay for candidates. But the most important unresolved question is the regulation of electoral expenses and other controls on political contributions and party financing.

* $\quad$ Professeur de droit constitutionnel, Faculté de droit, Université Laval à Québec; ce texte résulte d'une communication à la Table ronde internationale sur le droit de suffrage, Aix-en-Provence, 12 juillet 1989. 


\section{SOMMAIRE}

INTRODUCTION

1. LES TITULAIRES DU DROIT DE VOTE . . . . . . . 434

$1.1 \quad$ LES PRISONNIERS . . . . . . . . . . . . . . . . . 435

1.2 LES HANDICAPÉS MENTAUX . ............ 442

1.3 LES JUGES $\ldots \ldots \ldots \ldots \ldots \ldots \ldots \ldots \ldots, 443$

2. LES CONDITIONS D'EXERCICE DU SUFFRAGE ... 444

2.1 L'EXIGENCE DE RÉSIDENCE ............. 444

2.2 L'INSCRIPTION SUR LA LISTE ÉLECTORALE

446

2.3 LE VOTE DES ABSENTS $\ldots \ldots \ldots \ldots \ldots \ldots \ldots .448$

2.4 LA PERTE DU DROIT DE VOTE À TITRE DE

SANCTION PÉNALE $\ldots \ldots \ldots \ldots \ldots \ldots \ldots, 450$

3. LE DROIT D'ÉLIGIBILITÉ ET DE CANDIDATURE . 452

3.1 LE DROIT D'ÉLIGIBILITÉ EN GÉNÉRAL ...... 452

3.1.1 LES PARLEMENTAIRES ET LES JUGES.

3.1.2 LES FONCTIONNAIRES ET AUTRES AGENTS PUBLICS RELEVANT DU GOUVERNEMENT DU CANADA. ...... 453

3.1.3 LES FONCTIONNAIRES ORDINAIRES . 454

3.1.4 LES AUTRES AGENTS PUBLICS FÉDÉRAUX ................... 454

3.1.5 LES SHÉRIFS, GREFFIERS DE LA PAIX ET PROCUREURS DE LA COURONNE . 455

3.2 L'INÉLIGIBILITÉ POUR CAUSE DE RELATIONS CONTRACTUELLES AVEC LA COURONNE. ........................ 457

3.3 L'INÉLIGIBILITÉ À TITRE DE SANCTION PÉNALE ....................... 457

4. LES CONDITIONS DE VALORISATION DE L'EXPRESSION ÉLECTORALE . . . . . . . . . . . . . 459

4.1 L'ÉGALITÉ DE REPRÉSENTATION .......... 459

4.2 LE RÉGIME DES PARTIS POLITIQUES ....... 461

4.3 LE PLAFONNEMENT ET LE CONTRÔLE DES DÉPENSES ÉLECTORALES ................ 464

4.4 LE FINANCEMENT DES CANDIDATS ET DES

PARTIS ......................... 465 
(1991) 21 R.D.U.S.
La Charte constitutionnelle de 1982 et la démocratie électorale canadienne

4.4.1 L E $\quad$ F I N A N C E M E N T GOUVERNEMENTAL ........... 466

4.4.2 LES CONTRIBUTIONS POLITIQUES ... 467

4.5 LA PUBLICITÉ ÉLECTORALE . . . . . . . . . . 470

4.6 LA COMMUNICATION AUDIO-VISUELLE . . . 472

CONCLUSION .......................... 474 
La Charte constitutionnelle de 1982 et la démocratie électorale canadienne
(1991) 21 R.D.U.S. 


\section{INTRODUCTION}

Les quinze dernières années ont profondément marqué le droit électoral canadien. Avant l'entrée en vigueur de la Charte constitutionnelle de 1982, des réformes législatives importantes avaient considérablement modifié le cadre de l'expression électorale. Plus récemment, le législateur a également voulu revaloriser l'égalité de représentation par la redéfinition du découpage électoral. Quant à la Charte constitutionnelle, elle fait sentir son impact depuis 1982 en provoquant un élargissement des clientèles titulaires du droit de vote et une clarification des conditions d'exercice du suffrage.

Trois des dispositions de la Charte constitutionnelle sont au coeur de l'évolution du droit électoral, les articles 3, $2 b$ ) et 15 .

art. 3 «Tout citoyen canadien a le droit de vote et est éligible aux élections législatives fédérales ou provinciales.

art. 2 Chacun a les libertés fondamentales suivantes:

b) (..) liberté de pensée, de croyance, d'opinion et d'expression, y compris la liberté de la presse et des autres moyens de communication;

art. 15 (1) La loi ne fait acception de personne et s'applique également à tous, et tous ont droit à la même protection et au même bénéfice de la loi, indépendamment de toute discrimination (...).»

Les articles 2 et 15 peuvent faire l'objet d'une clause dérogatoire édictée par le législateur compétent, pour une période de cinq ans. Par ailleurs, les articles 2,3 et 15 peuvent faire l'objet de restrictions sous l'article 1 de la Charte mais «dans des limites qui soient raisonnables et dont la justification puisse se démontrer dans le cadre d'une société libre et démocratique», sous le contrôle du juge ${ }^{1}$.

Le processus électoral est le moyen privilégié pour une collectivité de tendre à l'idéal démocratique. On dira que le système électoral est démocratique si le plus grand nombre de citoyens ont le droit de vote et

1. Gérald A. Beaudoin «Les droits démocratiques», dans Gérald A. Beaudoin et Edward Ratushny (dir.), Charte canadienne des droits et libertés, $2^{\mathrm{e}}$ éd., Montréal, Wilson et Lafleur, 1989, p. 307-348. 
peuvent l'exercer dans des conditions favorables. Deux questions se soulèvent alors: qui sont les titulaires du droit de vote et quelles sont les conditions d'exercices du suffrage? On dira également qu'un système est démocratique si le plus grand nombre de citoyens aptes à ce faire peuvent se porter candidat afin de doter la collectivité d'élus représentatifs et compétents. Plusieurs questions se soulèvent alors: premièrement, la question du droit d'éligibilité et de candidature; deuxièmement, le rôle des partis politiques, ces intermédiaires devenus nécessaires au stade de développement actuel des démocraties occidentales; troisièmement, le rôle et la place de l'argent comme instrument susceptible de favoriser tantôt l'expression électorale; enfin, certains aspects de la liberté d'expression liés au processus électoral, tel le rôle des médias, la réglementation de la publicité, etc.

\section{LES TITULAIRES DU DROIT DE VOTE}

En 1982, suivant la Loi électorale du Canada ${ }^{2}$, avait qualité d'électeur pour l'élection des députés du Parlement du Canada tout citoyen canadien de 18 ans, mais était habile à voter tout électeur autre que les personnes suivantes: le directeur général et directeur général adjoint des élections, le président d'élection de chaque circonscription, les juges nommés par le gouverneur en conseil, les détenus des établissements pénitentiaires y purgeant une peine, les handicapés mentaux, les personnes déclarées inhabiles à titre de sanction pour manoeuvres frauduleuses ou actes illicites ${ }^{3}$.

Trois catégories particulières de personnes provoquèrent des contestations judiciaires en invoquant l'article 3 de la Charte: les détenus, les handicapés mentaux et les juges. Certaines législatures provinciales ont cependant pris les devants: c'est ainsi qu'en 1979, l'Assemblée nationale du Québec a déjà conféré le droit de vote aux prisonniers détenus tant dans les prisons provinciales que les pénitenciers fédéraux ${ }^{4}$. Quant aux juges, les lois électorales provinciales ne comportent généralement pas d'exclusion. Enfin,

2. Loi électorale du Canada, S.R.C. 1970 , c. 14 (1 ${ }^{\mathrm{er}}$ suppl.).

3. S.C. 1969-70, c.49, art 14(1),(4). Les dix provinces canadiennes ont des législations comparables pour l'élection à la législature provinciale. Au fédéral une importante réforme a été proposée en 1987 par le P.L. C-79, abandonné au feuilleton de la Chambre.

4. Loi électorale, L.Q. 1979, c.56: les pénitenciers qui reçoivent les personnes condamnées à une peine de deux ans et plus relèvent de la compétence fédérale. 
à partir de 1980, plusieurs législatures ont circonscrit l'exclusion du malade mental $^{5}$.

\subsection{LES PRISONNIERS}

Une bonne douzaine d'arrêts ont été rendus par diverses instances à partir de 1983. En 1983, la Cour d'appel de la Colombie-Britannique déclare que priver de leur droit de vote les détenus en probation sous le régime des libérations conditionnelles est contraire à la Charte ${ }^{6}$. Par contre, elle semble d'accord avec l'opinion émise par la Cour suprême de la ColombieBritannique $^{7}$ suivant laquelle la privation du droit de vote des détenus se justifie dans le cadre d'une société démocratique et n'est pas déraisonnable à cause des obstacles inhérents à l'incarcération: il en résulte que l'ensemble des contraintes imposées par le maintien de l'ordre et de la discipline carcérale rendent impossible le «free and democratic electoral choice contemplated by the Constitution» ${ }^{8}$. Il s'agit là de l'argument de base des tenants de l'interdiction.

En 1984, la Cour d'appel de la Saskatchewan se penche sur le dossier $^{9}$ et déclare inconstitutionnelle la privation du droit de vote des «prévenus», c'est-à-dire des personnes incarcérées en attente de leur procès ou du jugement final.

Quelques jours avant l'élection du 4 septembre 1984, un détenu réclame par voie d'injonction interlocutoire qu'on lui permette de voter en invoquant l'inconstitutionnalité de l'article 14 de la Loi électorale du Canada en regard de l'article 3 de la Charte. La Cour fédérale de première instance accorde l'injonction mais la Cour fédérale d'appel l'annule au motif que ce serait statuer provisoirement sur la validité constitutionnelle de la loi sans avoir procédé à une instruction complète ${ }^{10}$. La Cour suprême confirme ce jugement $^{11}$.

5. En 1980, au Manitoba, seuls les malades mentaux internés n'ont pas droit de vote. En 1984, en Ontario, seuls les malades mentaux internés qualifiés par décret. En 1988, en Colombie-Britannique, seuls les malades internés par ordonnance du tribunal.

6. Reynolds c. Colombie-Britannique (P.G.), [1983] 143 D.L.R. (3d) 365 (B.C.S.C.), (1984) 5 W.W.R. 270 (B.C.C.A.) Reynolds voulait poser sa candidature à une élection provinciale, ce qui impliquait le droit de vote.

7. Id., à la p. 273 (j. Nemetz).

8. Jolivet c. R. (1983), 7 C.C.C. (3d) 431, 436 (j. Taylor) (B.C.S.C.).

9. Maltby c. Saskatchewan (P.G.), [1984] 10 D.L.R. (4th) 745 (Sask. C.A.).

10. Gould c. Canada (P.G.), [1984] 1 C.F. 1119 (1 $1^{\text {re }}$ inst.), infirmé par [1984] 1 C.F. 1133 (C.F.A.).

11. [1984] 2 R.C.S. 124. 
En 1986, la Cour du Banc de la Reine du Manitoba déclare que la loi de cette province qui interdit le vote aux prisonniers viole l'article 3 de la Charte. Le juge estime que la prohibition absolue et totale ne répond pas au critère de proportionnalité ${ }^{12}$. Le juge refuse toutefois d'ordonner aux autorités la prise de mesures spéciales, telle l'installation de bureaux de scrutin dans les prisons. La Cour d'appel du Manitoba confirme ce jugement à cause des circonstances et de la proximité de l'élection ${ }^{13}$; en d'autres temps elle aurait été disposée à ordonner que des mesures soient prises en vertu de l'article 24 al.1 de la Charte pour rendre possible l'exercice du droit de vote.

En novembre 1985, des prisonniers demandent à la Cour fédérale d'ordonner aux autorités pénitentiaires de prendre des mesures précises pour permettre l'exercice du droit de vote que la Loi électorale du Québec leur confert. La Cour conclut qu'elle pouvait, sur la base de l'article 24 al. 1 de la Charte, émettre une ordonnance de mandamus même contre une autorité gouvernementale ou un ministre parce que la Charte établit un ordre nouveau ${ }^{14}$. Elle ordonne donc aux autorités pénitentiaires fédérales de permettre aux autorités provinciales, notamment le directeur général des élections du Québec, de préparer des bureaux de scrutin dans les pénitenciers.

En 1988, la Haute Cour de l'Ontario ${ }^{15}$ refuse de considérer comme inconstitutionnel l'article 14 de la loi électorale fédérale prétendant que le Parlement a voulu réserver la participation au processus démocratique aux seuls citoyens dignes et responsables. Selon le juge, l'exclusion des criminels est symbolique et renforce le concept de démocratie libérale responsable.

En 1988 également, un autre juge de la même Cour déclare que l'article 16 de la loi ontarienne, qui interdit le droit de vote aux détenus, est contraire à la Charte $^{16}$. Si la sanction des infractions criminelles et la protection de la société peuvent le justifier, en revanche, l'objectif de réhabilitation qui inspire la philosophie de notre système correctionnel plaide plutôt en faveur de la participation des détenus au processus électoral. Enfin, il y a, selon le juge, un aspect arbitraire dans le fait que les grands criminels sont traités sur le même pied que l'individu condamné à une semaine de prison pour une infraction statutaire.

\footnotetext{
12. Badger c. Manitoba (P.G.), [1986] 27 C.C.C. (3d) 158 (j. Scollin) (Man. Q.B.).

13. Badger c. Manitoba (P.G.) (1986), 32 D.L.R. (4th) 310 (Man. C.A.).

14. Levesque c. Canada (P.G.), [1986] 2 C.F. 287 (1 $1^{\mathrm{re}}$ inst.).

15. Sauvé c. Canada (P.G.) (1988), 66 O.R. (2d) 234 (j. Van Camp) (Ont. H.C.).

16. Grondin c. Ontario (P.G.) (1988), 65 O.R. (2d) 427 (j. Bowlby) (Ont. H.C.).
} 
Quelques jours avant l'élection fédérale de novembre 1988, un détenu demande à la Cour supérieure du Manitoba de déclarer inconstitutionnel l'article 14 de la loi électorale fédérale et d'ordonner au Directeur général des élections du Canada de prendre les mesures nécessaires pour l'exercice efficace du droit de suffrage. La Cour accèda à la demande, mais la Cour d'appel renversera ce jugement trois jours avant l'élection, soit le 18 novembre ${ }^{17}$. Les trois juges d'appel considèrent alors que l'interdiction de voter est justifiée par l'article 1 de la Charte. Pour le juge en chef Monnin il y a trois raisons: premièrement, la majorité des démocraties occidentales et presque la totalité des États américains limitent totalement ou largement ce droit; deuxièmement, donner le droit de vote aux prisonniers c'est en même temps leur donner le droit de candidature; troisièmement, de nombreuses personnes en déplacement perdent aussi leur droit de vote du fait qu'elle ne peuvent l'exercer. Un autre juge estime que du fait de l'emprisonnement, le détenu voit plusieurs de ses droits limités, notamment le droit à la mobilité de l'article 6, le droit à la liberté de l'article 7, le droit d'association de l'article 2(d); il s'agit là d'une conséquence inévitable, presque d'une limite inhérente, qui pourrait se justifier sans recourir à l'article 1 de la Charte.

La Cour d'appel du Manitoba est la dernière à s'être prononcée. Il est intéressant de relever à plusieurs reprises sous la plume des juges qu'il appartient avant tout au Parlement de régler cette question, qui devrait l'être uniformément dans les diverses provinces du Canada. Toutefois ce n'est pas là un argument très convaincant, sauf pour répondre à la première question du test de l'arrêt Oakes $^{18}$. À notre avis, la Cour n'a pas vraiment franchi toutes les étapes du test Oakes, le plus important du contrôle de constitutionnalité sous la Charte.

Comme nous l'avons déjà signalé, l'article 1 prévoit qu'il est possible d'apporter certaines restrictions aux droits et libertés garanties par la Charte. Celles-ci doivent cependant se justifier dans «le cadre d'une société libre et démocratique». La Cour suprême du Canada a déterminé dans l'arrêt Oakes le type de test à appliquer pour vérifier la compatibilité avec la Charte au regard de l'article 1. Dans l'arrêt $E d w a r d s^{19}$ elle a reformulé ce test et lui a donné une forme définitive.

17. Badger c. Canada (P.G.) (1988), 55 D.L.R. (4th) 177 (Man. C.A.) renversant le jugement du juge Hirschfield, non rapporté.

18. R. c. Oakes, [1986] 1 R.C.S.103 [ci-après Oakes].

19. Edward Books and Art Ltd. c. R., [1986] 2 R.C.S. 713; aussi Ford c. Québec (P.G.), [1988] 2 R.C.S. 712, 770. 
Lorsqu'il a été établi qu'une règle de droit restreint un droit protégé par la Charte, le gouvernement doit démontrer que la disposition vise des objectifs assez importants pour justifier une restriction à ce droit:

«En premier lieu, l'objectif que visent à servir les mesures qui apportent une restriction à un droit ou à une liberté garantis par la Charte, doit être "suffisamment important pour justifier la suppression d'un droit ou d'une liberté garantis par la Constitution" $\gg^{20}$.

\section{Qu'est-ce qui est «suffisamment important»?}

«Il faut à tout le moins qu'un objectif se rapporte à des préoccupations urgentes et réelles dans une société libre et démocratique, pour qu'on puisse le qualifier de suffisamment important ${ }^{21}$.

Une fois cette première étape franchie, le gouvernement doit démontrer le caractère raisonnable de la mesure contestée et sa justification. Il devra y avoir une certaine proportion entre la mesure adoptée et l'objectif recherché. Selon la Cour, ce critère de proportionnalité comporte trois volets:

«Premièrement, les mesures adoptées doivent être soigneusement conçues pour atteindre l'objectif en question. Elles ne doivent être ni arbitraires, ni inéquitables, ni fondées sur des considérations irrationnelles. Bref, elles doivent avoir un lien rationnel avec l'objectif en question. Deuxièmement, même à supposer qu'il y ait un tel lien rationnel, le moyen choisi doit être de nature à porter "le moins possible" atteinte au droit ou à la liberté en question: (...) Troisièmement, il doit y avoir proportionnalité entre les effets des mesures restreignant un droit ou une liberté garantis par la Charte et l'objectif reconnu "suffisamment important" $\gg^{22}$.

Dans l'esprit de la Cour suprême, plus l'atteinte portée au droit est sérieuse, plus l'objectif visé doit être important.

Quant à l'objectif poursuivi par le législateur, il n'est pas facile de le cerner avec précision. Suivant la jurisprudence citée, il s'agit de priver

20. Supra, note 18 à la p. 138 (les italiques sont du soussigné).

21. Id. à la p. 139; dans des arrêts plus récents, la Cour s'est satisfaite que le gouvernement établisse qu'il considère l'objectif important. Dans quelques 130 arrêts, la Cour a toujours conclu que l'objectif était important. 
de participation au processus démocratique ceux qui se sont mis au ban de la société par leurs actes criminels et de favoriser le maintien de l'ordre et de la sécurité dans les prisons. Ce sont certes des préoccupations importantes et réelles.

Y-a-t-il un lien rationnel entre l'interdiction de voter et l'objectif poursuivi? Comme certains juges l'ont relevé, il n'est guère rationnel sinon arbitraire de mettre sur le même pied les grands criminels et l'individu condamné à trois jours de prison pour défaut de payer une amende.

Même si l'on trouvait le lien rationnel, il est difficile d'admettre que le moyen porte le moins possible atteinte au droit en question: l'interdiction est en effet absolue, sans exceptions et sans nuances.

Enfin qu'en est-il de la proportionnalité entre les effets de la mesure et l'objectif? La mesure est évidemment radicale, mais l'objectif a une importance qui n'est pas absolue; elle est même controversée puisque certains prétendent qu'au contraire il faut faire participer les prisonniers au processus démocratique dans un but de réinsertion sociale et de réforme personnelle. Quant au maintien de l'ordre et de la sécurité dans les prisons, il s'agit d'un objectif atténué par le fait qu'il ne doit pas englober les impératifs de «commodité administrative». La Cour suprême dans l'arrêt $\operatorname{Singh}^{23}$ a, en effet, considéré que le Gouvernement ou l'Administration ne peut invoquer cet argument pour restreindre des droits protégés par la Charte.

À notre avis, l'interdiction absolue de voter ne peut passer le test de l'arrêt Oakes car c'est une mesure trop radicale qui viole l'article 3 de la Charte. Il en serait différemment si la perte de ce droit était reliée à la commission de certains crimes tel que la haute trahison ou la fraude électorale. Quant aux inconvénients que l'exercice du droit de vote cause aux autorités pénitentiaires, il n'a pas été démontré qu'ils sont insurmontables, du moins à l'égard de l'ensemble de la population carcérale. Par contre il n'est pas impensable que le législateur soit justifié de faire un régime particulier pour les criminels dangereux, ceux qui seraient susceptibles de profiter de l'occasion pour mettre en péril la sécurité de l'établissement ou du public. Il est toutefois possible que les circonstances entourant l'exercice du droit de vote comportent de tels dangers. 
Le législateur québécois ne semble pas réticent à se conformer aux exigences de la Charte. En 1980, l'Assemblée nationale du Québec avait donné le droit de suffrage à tous les détenus. En 1987, le Parlement fédéral étudiait le projet de loi C-79 au même effet.

\subsection{LES HANDICAPÉS MENTAUX}

La doctrine a mené le combat en faveur des handicapés mentaux surtout depuis les années 80 . Certains législateurs provinciaux ont alors commencé la libéralisation du droit de suffrage. Ainsi en 1980, le Manitoba limite l'exclusion aux malades internés dans un hôpital ou une institution pour handicapés mentaux. En 1984, l'Ontario n'exclut que les malades internés par ordre d'un tribunal.

La première contestation judiciaire a eu lieu au Manitoba en mars 1988. La Cour du Banc de la Reine déclare inconstitutionnelle l'article 31(b) de la loi manitobaine qui déniait le droit de vote aux patients des hôpitaux psychiatriques ${ }^{24}$. Puis en octobre 88 , la Cour fédérale est saisie d'une contestation de l'article 14(4) f) de la loi fédérale qui déclare inhabile à voter:

«Toute personne restreinte dans sa liberté de mouvement ou privée de la gestion de ses biens pour cause de maladie mentale.»

La Cour déclare que le droit de vote n'a pas de caractère absolu et pourrait être restreint lorsque les circonstances le justifient, mais qu'il est tout simplement erroné de présumer qu'une personne souffrant d'une incapacité mentale quelconque est incapable à tous points de vue, et notamment incapable de voter ${ }^{25}$.

L'interdiction est donc trop générale et disproportionnée par rapport à l'objectif recherché.

Dans plusieurs provinces, notamment au Québec depuis 1989, il n'y a plus aucune restriction au vote des malades mentaux, sauf ceux qui sont en curatelle. Le projet de loi C-79 du 30 juin 1987 était au même effet au niveau fédéral. Cette situation n'est toutefois pas entièrement

24. Canadian Mental Health Association c. Wallis and A.G. Manitoba, Man. Q.B. 17 mars 1988, n:Cl 88-01-27535 (j. Glowacki).

25. Conseil canadien des droits des personnes handicapées c. Canada, [1988] 3 C.F. 622, 625 (j. Reed). 
satisfaisante, pas plus que ne l'est l'interdiction du droit de vote aux personnes de moins de 18 ans. Cette dernière limitation, certes arbitraire, n'a pas été contestée mais pourrait l'être, car un tel mineur apte à exercer toutes sortes de responsabilités sociales ou économiques n'aurait pas l'aptitude à exercer adéquatement un choix démocratique éclairé. Pourquoi 18 ans et non 17 ou 16 ans?

La doctrine semble rejeter le critère de la capacité ou de l'aptitude à poser un geste lucide ${ }^{26}$. Le problème n'est pas pour autant résolu, car si l'on n'a aucun critère pour empêcher de voter ceux qui n'ont pas la lucidité voulue pour le faire, a-t-on vraiment permis l'exercice de ce droit? La solution se situe à notre avis à un double niveau. Premièrement, l'inscription sur la liste électorale devrait être plus rigoureuse et les agents électoraux ne devraient, en cas de doute, qu'inscrire ceux qui sont capables de faire une démarche lucide en ce sens ${ }^{27}$. D'autre part, toute personne inscrite sur la liste qui se présente au bureau de scrutin devrait voter sans assistance, sauf s'il s'agit d'handicapés physiques. Cela vaut non seulement pour les handicapés mentaux mais pour les personnes confuses pour raison d'âge ou autres. Il ne faut pas que le vote assisté devienne un vote téléguidé ou substitué.

\subsection{LES JUGES}

Assez étonnamment la loi électorale fédérale rendait inhabile à voter tout juge nommé par le gouvernement fédéral. Il s'agit d'une tradition reposant sur divers fondements, tels le principe de la séparation des pouvoirs, la dignité judiciaire,... Mentionnons que les lois provinciales ne comportent pas généralement une telle exclusion. Par ailleurs, la loi électorale fédérale ne vise pas les juges nommés par les gouvernements provinciaux qui sont pourtant des juges à part entière et sont assujettis aux mêmes exigences constitutionnelles d'indépendance ${ }^{28}$.

Cette disposition a été attaquée en Cour fédérale en novembre 1988 alors que le projet de loi C-79 qui l'abolissait était à l'étude devant le Parlement. Néanmoins la Cour fédérale déclara la disposition

26. Gérald B. Robertson, Mental Disability and the Law in Canada, Toronto, Carswell, 1987, pp. 240-250.

27. Le directeur général des élections n'a qu'à envoyer aux agents des directives en ce sens, comme cela s'est fait au Québec en 1989.

28. Voir notre étude: «La justice municipale au regard des chartes: quelques observations au lendemain de la grande réforme», [1991] 36 McGill L.J., pp. 39-76. 
inconstitutionnelle et la déclara inopérante pour permettre aux magistrats de voter à l'élection générale du 21 novembre ${ }^{29}$. Comme la requête n'était pas contestée, la décision n'est pas motivée.

\section{LES CONDITIONS D'EXERCICE DU SUFFRAGE}

Les lois électorales déterminent les diverses conditions d'exercice du droit de vote, notamment la question de la résidence qui a fait l'objet de contestations en regard de la Charte, de même que celle de la présence au lieu de résidence le jour du scrutin.

\subsection{L'EXIGENCE DE RÉSIDENCE}

La loi électorale fédérale n'exige aucune période de résidence préalable à l'inscription sur la liste électorale, mais plusieurs lois provinciales exigent une résidence de six ou douze mois. Cette exigence a fait l'objet de contestations.

En 1984, la Cour d'appel de la Saskatchewan ${ }^{30}$ avait à se demander si une exigence de six mois de résidence dans la province était contraire à la Charte. La Cour estime que le droit de vote n'étant pas absolu il peut être assujetti à certaines conditions dont la résidence. Mais encore faut-il que le délai soit raisonnable. Comment un électeur pourraitil apprécier les performances gouvernementales et les politiques proposées s'il ne connaît pas suffisamment le milieu. Or, six mois constitue une période raisonnable... d'autant plus que dans d'autres provinces elle est soit plus longue soit de même longueur.

En 1986, la Cour d'appel du Yukon était saisi d'un renvoi dans lequel le gouvernement de ce territoire demandait à la Cour quel délai de résidence était compatible avec la Charte: un an, six mois ou un délai minimum à des fins administratives ${ }^{31}$. Ce renvoi était consécutif à une décision de la Cour supérieure du Yukon qui avait déclaré l'exigence de 12 mois alors en vigueur contraire à l'article 3 de la Charte $^{32}$. Le juge Nemetz au nom de la Cour d'appel estime que dans ces territoires aux caractéristiques particulières il n'est pas déraisonnable d'exiger une

29. Muldoon c. Canada, [1988] 3 C.F. 628 (j. Walsh) (1 $1^{\text {re }}$ inst.).

30. Re Storey and Zazelenchuk (1984), 12 C.R.R. 261 (Sask. C.A.), confirmant (1982), [1984] 5 C.R.R. 99 (j. Estey).

31. Re Yukon Election Residency Requirement, [1986] 27 D.L.R. (4th) 146 (Y. C.A.)

32. Hedstrom c. Com'r of Yukon Territory, [1985] 6 W.W.R. 690 (j. Maddison). 
période plus longue de résidence. S'appuyant sur le constitutionnaliste américain Tribe, il conclut qu'il appartient à une collectivité de définir son identité et le type de lien qu'elle souhaite entre les élus et le corps électoral. Ainsi seront électeurs ceux qui ont «a real nexus to the community as such ${ }^{33}$.

La Cour d'appel du Yukon considère que l'objectif du législateur dans la fixation d'une exigence de résidence est triple: l'assurance de l'intégrité du processus électoral; l'assurance que les voteurs sont adéquatement informés des enjeux électoraux et l'assurance que les voteurs ont une appartenance territoriale suffisante. Par ailleurs, la mobilité inhérente à un État fédéral et les fluctuations de population justifient de la part des législatures provinciales une telle exigence. La Cour insiste sur l'idée d'appartenance au milieu pour donner un sens au processus démocratique. Le vote n'est pas qu'un choix pour l'avenir, c'est un jugement sur la performance passée d'un gouvernement, qu'un nouvel arrivant aurait du mal à formuler. Enfin, la Cour ajoute que celui qui n'est pas suffisamment familiarisé avec les conditions locales peut mettre en danger l'intégrité du processus électoral, notamment en se laissant aller à des pratiques électorales douteuses.

Il semble bien qu'un délai de résidence raisonnable de six mois ou même de douze mois soit considéré, au Canada, comme compatible avec l'article 3 de la Charte. Il nous semble que cela se justifie si l'on cherche à donner à l'exercice du droit de suffrage un sens véritable. À cet égard, on pourrait distinguer entre la résidence dans la province et la résidence dans la circonscription; le délai de résidence dans la circonscription pourrait être plus court. De façon générale, les lois provinciales s'alignent presque toutes sur un délai de résidence de six mois, ce qui nous paraît fort acceptable. 
La Charte constitutionnelle

de 1982 et la démocratie

(1991) 21 R.D.U.S.

électorale canadienne

\subsection{L'INSCRIPTION SUR LA LISTE ÉLECTORALE}

Toutes les lois exigent qu'outre la question de résidence, l'électeur soit inscrit sur la liste électorale avant le jour du scrutin; toutefois certaines prévoient que, dans certaines circonstances, un électeur puisse voter sans être inscrit, soit en étant assermenté. Ainsi au niveau fédéral, la loi offre cette possibilité mais aux électeurs des districts ruraux seulement. Ces dispositions ont été contestées en 1988 par des électeurs de districts urbains ${ }^{34}$. La Haute Cour de l'Ontario considéra que le droit consacré par l'article 3 de la Charte n'étant pas absolu, l'exigence de l'inscription obligatoire sur la liste électorale n'était pas déraisonnable; l'absence d'une telle mesure rendrait la gestion électorale extrêmement complexe dans les milieux urbains notamment. Par ailleurs, la Cour estima que l'avantage conféré aux électeurs ruraux ne constituait pas une discrimination ou un manquement au principe d'égalité consacré par l'article 15 de la Charte.

La Cour suprême de Colombie-Britannique fut saisie d'une contestation identique et, dans une décision du 28 juin 1990, considère que les articles 120 et 121 de la loi électorale fédérale ne violent pas l'article 15 de la Charte ${ }^{35}$. Se fondant sur l'arrêt Turpin $^{36}$ de la Cour suprême, la Cour refuse de considérer les électeurs urbains comme une «discrete and insular minority»: ces personnes ne font pas l'objet d'une discrimination

«from other persons on the basis of a personal characteristic which shares the similarities of historical, social, legal or political desavantage as those enumerated in $5.15^{37}$.

L'inscription obligatoire sur la liste électorale est certes une exigence très largement répandue. Mais le fait de ne prévoir aucune exception dans les milieux urbains constitue-t-il une mesure trop radicale, disproportionnée par rapport à l'objectif visé par le législateur? Certes le processus de recensement des électeurs et de confection de la liste électorale est adéquat; mais il se trouvera toujours des électeurs qui, pour des raisons de force majeure, n'ont pu être recensés et n'ont pu demander

34. Koutroulides c. Canada (P.G.) (1988), 67 O.R. (2d) 655 (j. Hollingworth).

35. Scott c. Chief Electoral Officer for Canada, B.C. S.C. 28/06/90, [1990] B.C.D. Civ. 1230-01, J. Coultas.

36. R. c. Turpin, [1989] 1 R.C.S. 1296.

37. Supra, note 34 . 
une révision dans les délais. Dans l'affaire Koutroulides $^{38}$, le juge ontarien se contente de présumer que l'admission d'une telle exception causerait «some real difficulties», mais il avoue qu'on n'en a pas fait la démonstration. À notre avis, il appartenait au gouvernement de faire cette démonstration. Tant que le gouvernement n'aura pas démontré que l'admission d'une telle exception perturbe considérablement le processus électoral, à notre avis, l'article 39(1) de la loi fédérale est inconstitutionnelle, au regard de l'article 3 de la Charte, car il limite l'exercice du droit de vote. Par contre, nous estimons qu'il n'y a pas violation de l'article 15 pour les raisons données par la Cour de Colombie-Britannique.

\subsection{LE VOTE DES ABSENTS}

Le vote par anticipation permet aux électeurs qui seront absents le jour du scrutin d'exercer leur droit de vote; toutefois, bon nombre d'électeurs ne peuvent même pas participer à ce vote par anticipation qui a lieu dans la circonscription une semaine avant le scrutin. La loi électorale fédérale prévoit le vote par procuration pour diverses catégories de personnes. D'autres lois provinciales, dont celle du Québec en 1989, prévoient la possibilité pour les électeurs absents d'exercer ce droit. Cette question est loin d'avoir été complètement résolue au Canada dans son ensemble.

En 1986, la Cour d'appel de la Colombie-Britannique a été saisie d'une affaire dans laquelle deux étudiants, pourtant inscrits sur la liste électorale, ne pouvaient se présenter au bureau de scrutin du fait de leurs études en Ontario ${ }^{39}$. Ils réclamèrent que la loi électorale soit déclarée non conforme à l'article 3 de la Charte et que soit ordonné à la législature de prévoir des dispositions pour permettre le vote des absents.

La Cour fit retrancher la conclusion dirigée contre la législature mais accepta de prononcer un jugement déclaratoire. Elle soumit qu'il importait peu que la privation du droit de vote résulte d'une disposition expresse ou d'une carence de la législation. Elle rappelle que la majorité des lois électorales canadiennes prévoient le vote des absents par procuration; de même, un rapport de la Colombie-Britannique de 1978 a recommandé de modifier la loi en ce sens. Elle conclut qu'on n'a pas 
démontré que l'omission du législateur était raisonnable et justifiable sous l'article 1 de la Charte.

Le procédé de la Cour d'appel de la Colombie-Britannique est peut-être peu orthodoxe, mais le résultat est intéressant. On peut dire que, dans l'ensemble, les législateurs ont pris leurs responsabilités en ce domaine, mais la Charte n'a pas nuit.

Dans une affaire plus récente, un ancien résident de l'Ontario, absent de sa province pendant une longue période, prétendait que l'exigence de six mois de résidence en Ontario contrevenait à l'article 3 de la Charte en lui faisant perdre son droit de vote ${ }^{40}$. Il prétendait que dans son cas le délai était déraisonnable. La Cour rejeta le pourvoi en estimant que le législateur avait fixé une limite raisonnable au droit consacré par la Charte. Il appartient au législateur de tirer la ligne, comme l'ont estimé les Cours d'appel de la Saskatchewan et du Yukon dans les arrêts précités. La cour n'a pas à tenir compte des situations individuelles qui peuvent varier à l'infini.

Enfin, en 1986 était contestée une disposition de la loi de la Colombie-Britannique qui conférait à une personne dont le nom n'apparaissait sur la liste d'aucune circonscription électorale le droit de se faire inscrire le jour du vote ${ }^{41}$. Or, la personne inscrite dans une circonscription qui subséquemment déménage dans une autre circonscription ne peut bénéficier du même avantage; elle doit se faire réinscrire suivant la procédure et dans le délai régulier, c'est-à-dire 20 jours avant la date du scrutin. Le requérant prétendait que cette dernière exigence faisait perdre à l'électeur qui déménage durant cette période de 20 jours son droit de vote. De plus, il prétendait que la disposition était discriminatoire et contraire au droit à l'égalité consacré par l'article 15 de la Charte.

La Cour suprême de la Colombie-Britannique rejeta les deux allégations. Premièrement, le délai de 20 jours n'est pas déraisonnable suivant les exigences d'une procédure normale. De plus, l'électeur peut exercer son droit de vote par procuration dans la circonscription où il résidait à la date du recensement. Quant au désavantage qui résulte de la disposition contestée, elle ne constitue pas une «discrimination» au sens

40. $\quad$ Arnold c. Ontario (P.G.) (1987) 61 O.R.(2d) 481.

41. Re Scott and A.G. B.C., [1986] 29 D.L.R (4th) 544 (B.C. S.C.). 
où la jurisprudence l'a reconnu sous l'article 15 de la Charte. La Cour estime que le législateur doit se plier à certaines contraintes qui ont pour effet de restreindre l'exercice du droit de vote, la mobilité de la population dans la province en est une ${ }^{42}$. Le législateur devrait-il permettre la réinscription dans une nouvelle circonscription et l'annulation de la première inscription après un déménagement qui survient dans les 20 jours précédant l'élection? C'est là beaucoup demander. Il appartient au législateur et non au juge de trouver un moyen.

\subsection{LA PERTE DU DROIT DE VOTE À TITRE DE SANCTION PÉNALE}

On peut considérer comme une condition d'exercice du suffrage le fait d'en être privé par condamnation pénale spécifique, tel le cas de fraude électorale. Au Canada, toutes les lois électorales prévoient une telle sanction. Ainsi l'article 51(g) de la loi fédérale stipule que ne peut voter «toute personne inhabile à voter en vertu d'une loi relative à la privation du droit de vote pour manoeuvres frauduleuses ou actes illégaux». Cette disposition renvoie aux articles 267 et suivants de la $1 \mathrm{loi}^{43}$.

Si l'infraction constitue une manoeuvre frauduleuse (art. 250, 252, 253 ou 260), la perte du droit de vote est de sept ans; s'il s'agit d'une autre infraction à la loi électorale, elle est de cinq ans (art. 269).

Puisqu'il s'agit d'une restriction au droit protégé par l'article 3 de la Charte, il faut appliquer le test Oakes pour vérifier si, au regard de l'objet et de la nature du droit constitutionnalisé, l'atteinte est raisonnable et justifiable dans le cadre d'une société libre et démocratique.

Premièrement, l'objectif du législateur est certes «suffisamment important» en ce sens qu'il correspond à des «préoccupations urgentes et réelles». Le législateur a comme objectif d'assurer l'intégrité du processus électoral et de permettre l'élection des personnes les plus aptes à exercer la fonction parlementaire.

Deuxièmement, la mesure adoptée est-elle proportionnée à l'objectif recherché? Le critère de proportionnalité comprend trois volets.

\footnotetext{
42. Dans cette décision on mentionne qu'à l'élection provinciale de 1985, un demi-million d'électeurs ne s'étaient pas inscrits lors du recensement.

43. Loi électorale du Canada, S.R.C. 1970, c. 14 (1 ${ }^{\mathrm{er}}$ suppl.) est devenu l'art. 51 dans S.R.C. 1985 , c. E-2.
} 
Primo, y-a-t-il un lien rationnel avec l'objectif en question? Secondo, le moyen choisi est-il de nature à porter le moins possible atteinte au droit protégé? Tertio, y-a-t-il disproportion entre les effets de la mesure et l'objectif reconnu «suffisamment important».

Il y a manifestement un lien rationnel avec l'objectif visé puisqu'il s'agit de sanctionner des infractions à la loi électorale. Toutefois, il n'est pas possible de soutenir que le moyen choisi porte le moins possible atteinte au droit de vote; il le supprime, alors qu'il existe d'autres moyens moins draconiens tels l'amende ou l'emprisonnement. Quand à la proportionnalité entre les effets de la mesure et l'objectif, il y aurait peutêtre lieu de faire une distinction entre les infractions graves que sont les manoeuvres frauduleuses qui affectent gravement l'intégrité du processus électoral et d'autres infractions qui peuvent n'avoir qu'un caractère technique, tel par exemple le fait de publier, distribuer ou afficher un imprimé sans indiquer l'autorisation requise (art. 261 par. 2). Le droit de vote est certes le droit le plus fondamental de la structure politique canadienne, tellement qu'il ne peut être affecté par une clause nonobstant ou dérogatoire; aussi, seule une infraction d'une exceptionnelle gravité peut justifier une limitation. $\grave{A}$ notre avis, seules les manoeuvres électorales frauduleuses devraient être retenues pour légitimer la suppression du droit de vote pendant une période raisonnable, qui peut être de 5 ou 7 ans.

La loi québécoise de $1989^{44}$ nous semble s'être engagée dans la bonne voie. Elle distingue les manoeuvres électorales frauduleuses et les autres infractions, et donne, dans un cas donné, discrétion au juge de qualifier une infraction de manoeuvre frauduleuse. Seules ces dernières font encourir, par l'effet de la loi, la perte du droit de vote de même que le droit de se livrer à du travail partisan ou d'être candidat, pour une période de cinq ans (art. 568).

\section{LE DROIT D'ÉLIGIBILITÉ ET DE CANDIDATURE}

L'article 3 de la Charte de même que notre tradition législative semblent lier le droit d'égibilité au droit de vote. Mais le rapport entre les deux droits n'est pas constant; et il pourrait se différencier davantage à partir du moment où s'élargit le droit de vote à des titulaires qui pourraient raisonnablement se voir dénier celui de se porter candidat. On peut 
estimer qu'il faut, pour être député ou parlementaire, un niveau de qualification différent de celui exigé du simple électeur.

\subsection{LE DROIT D'ÉLIGIBILITÉ EN GÉNÉRAL}

En vertu de la loi fédérale actuelle, tous ceux qui ont la qualité d'électeur, c'est-à-dire les citoyens canadiens ayant atteint l'âge de 18 ans, ont droit d'éligibilité à moins qu'ils ne soient inhabiles à voter suivant l'article 51 ou qu'ils ne soient déclarés inéligibles suivant l'article 77 . Il y a donc de nombreuses restrictions au droit d'éligibilité consacré par l'article 3 de la Charte. Chacune de ces restrictions est-elle raisonnable et justifiable dans le cadre d'une société libre et démocratique?

\subsubsection{LES PARLEMENTAIRES ET LES JUGES}

L'article 77(d) et (g) prévoit expressément le cas des députés des provinces, des Territoires du Nord-Ouest et du Yukon. Le cas des sénateurs n'est pas expressément prévu. Quant aux juges, ils étaient inéligibles par le biais de l'art. 77(h) et 51(d), cette dernière disposition a été déclarée inconstitutionnelle en 1988 dans l'arrêt Muldoon ${ }^{45}$.

L'inéligibilité des parlementaires et des juges se justifie amplement par le principe de la séparation des pouvoirs, principe constitutionnel fondamental qui est l'assise de tout notre édifice constitutionnel. La loi devrait le dire expressément et inclure, à notre avis, tous les juges rattachés à la branche judiciaire de l'État, qu'ils soient nommés par le gouvernement fédéral ou par les provinces. Les juges provinciaux des cours ordinaires de justice ont une mission capitale dans l'interprétation et l'application des lois tant provinciales que fédérales; même les juges municipaux sont appelés à appliquer le code criminel. Il nous semble que l'incompatibilité se justifie amplement.

\subsubsection{LES FONCTIONNAIRES ET AUTRES AGENTS PUBLICS RELEVANT DU GOUVERNEMENT DU CANADA}

L'article 77(f) déclare inéligibles tous les agents publics relevant du gouvernement fédéral et dont la nomination relève de la Couronne, qu'il s'agisse d'une charge ou d'un emploi permanent ou temporaire, mais 
l'article 78(g) redonne l'éligibilité à tous les fonctionnaires régis par la Loi sur l'emploi dans la fonction publique ${ }^{46}$ pourvu qu'ils aient obtenu un congé sans solde suivant l'article 33 de cette dernière loi, c'est-à-dire avec l'autorisation de la Commission de la fonction publique.

\subsubsection{LES FONCTIONNAIRES ORDINAIRES}

Ce qui pose difficulté, comme nous l'avons expliqué dans une autre étude ${ }^{47}$, c'est que le refus d'un congé sans solde puisse être considéré comme un obstacle à la réalisation d'un droit constitutionnel consacré à l'article 3 de la Charte. Nous avons conclu que le congé sans solde devrait être accordé de plein droit. Mais, la réintégration dans la fonction publique au terme du congé électoral sans solde peut donner lieu à une évaluation des aptitudes et capacités, par la Commission de la fonction publique en vue de l'attribution d'un poste, en tenant compte des exigences d'efficacité et des objectifs du législateur, qui sont de favoriser l'exercice des droits politiques de tous les citoyens y compris les fonctionnaires. La Cour supérieure de la Nouvelle-Écosse en est arrivé à la même conclusion dans un arrêt de $1986^{48}$.

\subsubsection{LES AUTRES AGENTS PUBLICS FÉDÉRAUX}

L'incompatibilité est ici absolue, en ce sens que les titulaires de ces postes doivent démissionner purement et simplement sans possibilité de congé électoral.

Si l'on applique à ces personnes les critères utilisés pour l'ensemble des fonctionnaires, il faudrait pouvoir leur appliquer des règles d'intégration appropriées. Se soulève alors diverses difficultés. D'un côté, s'il s'agit d'agents nommés pour une période fixe ou suivant bon plaisir, la réintégration dans la fonction publique régulière équivaudrait à leur

46. Loi sur l'emploi dans la fonction publique, S.R.C. 1985, c. P-33.

47. «La liberté politique des fonctionnaires à l'heure de la Charte canadienne», (1990) 31 $C$. de D., 409-476; nous y traitons également des limites à la liberté d'expression politique des fonctionnaires en période électorale.

48. Re Fraser and A.G. Nova Scotia (1986) 30 D.L.R. (4th) 430. Dans un arrêt de 1988, la Cour de l'Ontario estimait que la question des congés électoraux n'avait rien à voir avec le droit d'éligibilité protégé par la Charte: O.P.S.E.U.C. Ontario (P.G.) (1988), 65 O.R. (2d) 689. Dans la plupart des provinces, les fonctionnaires ordinaires ont droit à un congé électoral sans solde prévu expressément par la loi. La loi québécoise de 1989 va d'ailleur plus loin en créant ce droit en faveur de tout employé du secteur public ou privé (art. 248-255). 
octroyer la permanence au retour d'un congé électoral: c'est le cas des membres de commissions, offices, bureaux, tribunaux administratifs ou autres organismes autonomes. Il ne saurait être question que ces personnes réintègrent leur poste après avoir plongé dans l'action politique électorale, à moins d'être de nouveau nommées suivant la procédure régulière.

Le cas des sous-ministres ou sous-chefs qui ont le titre de fonctionnaire, est différent vu qu'ils ont déjà la permanence dans la fonction publique. La réintégration serait alors possible dans un autre poste après évaluation par la Commission de la fonction publique. Ainsi avons nous conclu dans l'étude précitée qu'il est possible de leur appliquer le même régime qu'aux autres fonctionnaires. Il s'agit d'une solution qui rend possible l'exercice du droit de candidature sans inconvénients majeurs pour l'appareil gouvernemental et sans atteinte aux principes d'indépendance, de neutralité et d'impartialité de la fonction publique.

\subsubsection{LES SHÉRIFS, GREFFIERS DE LA PAIX ET PROCUREURS DE LA COURONNE}

Cette catégorie d'agents publics, fonctionnaires provinciaux pour la plupart, exercent des fonctions qui les relient d'assez près au pouvoir judiciaire, mais ce ne sont pas des juges. L'inéligibilité décrétée par l'article 77(e) ou son équivalent, a été étudiée par la Commission de la fonction publique du Québec dans une décision de $1989^{49}$. La Commission a conclu qu'une telle inéligibilité n'était pas déraisonnable et se justifiait dans le cadre d'une société libre et démocratique. Il faut toutefois signaler que le gouvernement avait offert au substitut du Procureur général visé par la mesure de congédiement de le réintégrer dans la fonction publique dans un autre poste de conseiller juridique.

La Commission qualifie la fonction de procureur de la Couronne de «quasi-judiciaire». Puis elle soutient que la disposition législative a pour objet de promouvoir le maintien de l'autorité, de la neutralité, de

49. Tremblay et Ministère de la Justice du Québec (1989), 6 R.D.C.F.P. 145 (Commissaire Roberge). M. Tremblay a été candidat N.P.D. dans Rivière-Du-Loup à l'élection fédérale de 1988: l'article 8 de la loi québécoise sur les substituts du Procureur général interdit à cette catégorie de fonctionnaires «de se porter candidat à une élection fédérale ou provinciale». Il fut de ce fait destitué. Devant la Commission, il invoqua en outre la Loi sur la fonction publique en vertu de laquelle il aurait eu droit à un congé électoral s'il avait été un fonctionnaire ordinaire. 
l'impartialité réelle et apparente et de l'intégrité du système judiciaire, en particulier en matière de justice criminelle et pénale.

Quant à la nature des fonctions exercées, la Cour suprême s'est depuis de nouveau prononcée en la qualifiant de quasi-judiciaire ${ }^{50}$; toutefois, cela ne saurait valoir pour les shérifs et greffiers de la paix. On ne peut mettre en doute l'importance de l'objectif du législateur, mais la mesure est-elle disproportionnée ou trop radicale? La Cour supérieure a renversé la décision de la Commission et invalidé l'article 8 de la Loi sur les substituts du procureur général comme contraire à l'article 3 de la Charte et discréminatoire en regard de l'article 15 de la même Charte ${ }^{51}$. La Cour estime que cette restriction au droit de candidature et au droit à l'égalité, car la Loi sur la fonction publique confert le droit à un congé électoral à l'ensemble des fonctionnaires, ne se justifie pas sous l'article 1 de la Charte!

La solution qui respecterait l'esprit de la Charte serait que le législateur prévoit un congé électoral avec droit de réintégration dans la fonction publique à un poste autre que celui de procureur de la Couronne.

\subsection{L'INÉligibILITÉ POUR CAUSE DE RELATIONS CONTRACTUELLES AVEC LA COURONNE}

L'article 77(c) de la loi fédérale déclare inéligible toute personne qui, directement ou indirectement, par elle-même ou par personne interposée a un contrat avec le gouvernement pour lequel des deniers publics doivent être versés; cela ne comprend cependant pas l'actionnaire de sociétés commerciales sauf si le contrat vise la construction d'un ouvrage public.

Il s'agit d'une restriction au droit d'éligibilité destiné à prévenir les conflits d'intérêts et la corruption politique. L'idée qu'un député ait des relations contractuelles avec la Couronne répugne à l'idée de moralité publique. Le député participe au vote des budgets nécessaires à l'activité contractuelle du gouvernement; d'autre part, il participe au contrôle de l'activité gouvernementale qui comprend bien entendu d'innombrables

50. Nelles c. Ontario, [1989] 2 R.C.S. 170.

51. Tremblay c. Commission de la fonction publique, J.E. 90-888 (C.S.). 
contrats. Il est raisonnable et justifiable dans une société démocratique que le député ne soit pas impliqué dans ces contrats de manière à être entièrement objectif et désintéressé dans son travail.

\subsection{L'INÉLIGIBILITÉ À TITRE DE SANCTION PÉNALE}

L'article 77 (a) et (b) prévoit des inéligibilités pour des périodes de cinq et sept ans à l'encontre d'une personne condamnée judiciairement pour manoeuvre électorale frauduleuse ou autre pratique illégale reliée à une élection. Une disposition analogue a été contestée devant la Cour suprême de la Nouvelle-Écosse dans la célèbre affaire MacLean ${ }^{52}$.

La disposition contestée prévoyait qu'une personne condamnée pour une infraction punissable par un emprisonnement de plus de cinq ans est déchue de ses droits d'éligibilité pour une période de cinq ans; s'il s'agit d'un député en poste, son siège devient vacant... La juge en chef de la Cour suprême de la Nouvelle-Ecosse commence par mettre en doute l'objectif même du législateur: il s'agit d'une législation «protectrice» de l'électorat, mais on devrait plutôt donner le crédit à ce dernier de faire son propre choix judicieux: «The legislation is paternalistic and excessive.... ${ }^{53}$. Dans un deuxième temps, elle considère la loi «under the proportionality test... unnecessary to protect society» ${ }^{54}$. Elle ajoute que les critères retenus par le législateur éliminent les personnes condamnées pour des infractions qui n'ont rien à voir avec ce qu'elle appelle «breaching the trust of the House» ${ }^{55}$. L'arrêt n'est pas très bien articulé et sa portée est à notre avis limitée. Serait contraire à la Charte toute disposition prévoyant l'inéligibilité à titre de sanction sans que l'auteur soit impliqué dans un «breaching the trust of the House», c'est-à-dire une infraction qui se rapporte au processus démocratique ou électoral. Il s'agit d'une infraction par laquelle le contrevenant s'est montré indigne de la confiance de la législature. Voilà un critère qui nous paraît valable. $\mathrm{Ce}$ serait le cas, par exemple, des manoeuvres électorales frauduleuses de même que des infractions criminelles impliquant fraude ou corruption

52. MacLean c. Nouvelle-Écosse (P.G.), [1987] 76 N.S.R. (2d) 296 (j. Glube). Billy Joe MacLean avait été condamné pénalement et civilement en justice pour avoir présenté pour $\$ 22,000$ de faux comptes de dépenses. Comme il refusait de démissionner il fut expulsé par une loi spéciale de la législature. Il fut réelu quelques semaines après l'arrêt de la Cour.

53. II. à la p. 306.

54. Id.

55. $I d$. 
politique. En commettant ces infractions, une personne s'est montrée indigne de briguer les suffrages.

Quant à l'argument de la juge en chef à l'effet que l'électorat n'a pas besoin d'être protégé, il nous parait discutable. Le législateur peut prendre des mesures en vue de favoriser un choix éclairé entre candidats dignes d'être investis de la fonction législative.

À notre avis, le respect intégral de la loi électorale de même que des dispositions du Code criminel concernant la corruption politique devrait être exigé de tous ceux qui briguent les suffrages. L'inéligibilité qui résulte de la violation de ces dispositions est à notre avis raisonnable et justifiable dans le cadre d'une société libre et démocratique.

\section{LES CONDITIONS DE VALORISATION DE L'EXPRESSION ÉLECTORALE}

Les lois contemporaines ont imaginé des moyens ou techniques qui n'existaient pas à l'aube de la démocratie parlementaire, afin de valoriser l'expression électorale en éliminant des obstacles de tout ordre et en fournissant des moyens qui, dans certains cas, peuvent rétablir l'égalité entre les participants. On retrouve des dispositions législatives concernant les partis politiques, le financement des dépenses électorales, le plafonnement de ces dépenses, les contributions électorales, la réglementation de la publicité et de la communication audio-visuelle. Toutefois, la première condition de valorisation de l'expression démocratique consiste en l'égalité de représentation, question vivement débattue dans la jurisprudence américaine depuis plus de $30 \mathrm{ans}^{56}$.

\subsection{L'ÉGALITÉ DE REPRÉSENTATION}

La participation démocratique serait faussée si chaque vote n'avait pas le même poids dans la balance. Toutefois, la tradition jurisprudentielle et législative est à l'effet que cette égalité n'a pas qu'une dimension quantitative; elle a aussi une dimension qualitative en ce sens que sont également pris en compte des intérêts communautaires variables. De toute façon, un découpage des circonscriptions qui viserait l'égalité quantitative absolue est pratiquement impossible. La situation peut, en

56. Voir Lawrence H. Tribe, American Constitutional Law, $2^{\mathrm{e}}$ éd., Mineola, N.Y., Foundation Press, 1988, pp. 1063-1074; les arrêts principaux ont été Baker c. Carr, 369 U.S. 186 (1962), Reynolds c. Sims, 377 U.S. 533 (1964), etc. 
plus, se compliquer au niveau d'une législature fédérale à cause de certains impératifs constitutionnels. Ainsi, la loi fédérale stipule que la délimitation des circonscriptions repose sur la répartition de la population correspondant «dans la mesure du possible» au quotient obtenu en divisant par le nombre de sièges à pourvoir. Les écarts sont possibles en prenant en considération les éléments suivants:

«(i) la communauté d'intérêts ou l'identité culturelle d'une circonscription électorale d'une province ou son évolution historique,

(ii) le souci de faire en sorte que la superficie des circonscriptions dans les régions peu peuplées, rurales ou septentrionales de la province ne soit pas trop vaste ${ }^{57}$.

Sauf dans des circonstances considérées «comme extraordinaires» les écarts n'excèdent pas vingt-cinq pour cent.

Si l'on retrouve des variations importantes à l'intérieur de chacune des provinces, ces variations s'accentuent entre les provinces de telle sorte que ces distortions feront vraisemblablement l'objet de contestations ${ }^{58}$.

La seule loi canadienne sur le découpage des circonscriptions à avoir été attaquée depuis la Charte est celle de la Colombie-Britannique ${ }^{59}$. La Cour suprême de cette province a déclaré ces dispositions contraires à l'article 3 de la Charte qui implique l'égalité du pouvoir de vote ${ }^{60}$. Toutefois, l'article 3 ne requiert pas l'égalité absolue; depuis 1867 au Canada, on a toujours assoupli l'égalité de représentation en tenant compte de facteurs géographiques et d'intérêts régionaux. Depuis la Charte on doit considérer que l'égalité est un idéal qui peut admettre des écarts justifiés:

«on the ground that they contribute to better government of the populace as a whole, giving due weight to regional issues within

\footnotetext{
57. Loi sur la révision des limites des circonscriptions électorales, S.R.C. 1985, c. E-3, modifié par c.6 ( $2^{\text {ième }}$ supp.), art. 2 b).

58. John C. Courtney, «Parliament and Representation: The Unfinished Agenda of Electoral Redistributions», (1988) 21 Rev. Can. de Sc. Pol. 675.

59. Sur l'ensemble, voir: Alan Stewart, «Constitutional Law-Right to Vote - RedistributionEquality of Representation», (1990) 69 R. du B. Can. 355.

60. Dixon c. British Columbia (P.G.), [1989] 59 D.L.R. (4th), 247 (j. McLachlin).
} 
the populace and geographic factors within the territory government» ${ }^{61}$.

La Cour conclut que la loi contestée ne respectait pas ces principes. De façon générale, la majorité des lois canadiennes admettent un écart de plus ou moins $25 \%$. Or, le résultat obtenu par l'application de la loi donnait à l'une des circonscriptions 15 fois plus de voteurs que la plus petite d'entre elles. On trouvait aussi des écarts allant de $-86.8 \%$ à $+63.2 \%$ de la moyenne provinciale. Des considérations géographiques ou régionales ne peuvent expliquer tous ces écarts même en ColombieBritannique.

L'intérêt de cette première décision canadienne sur la question est qu'elle précise les exigences de l'article 3 de façon relativement souple, en affirmant qu'il appartient au législateur de faire ses choix. Cependant, le juge interviendra au nom de la Charte si les écarts ne se justifient pas par des considérations de représentation d'intérêts régionaux ou particuliers. La tâche de la Cour était d'autant plus facile, en l'espèce, qu'une Commission d'enquête avait en 1988 proposé une réforme qui respectait ces exigences.

\subsection{LE RÉGIME DES PARTIS POLITIQUES}

L'encadrement législatif de l'existence et du fonctionnement des partis, surtout en période électorale, concerne les articles 2, 3 et 15 de la Charte. Les réglementations trop rigoureuses peuvent gêner l'exercice du droit d'éligibilité, l'exercice des libertés d'opinion et d'expression, et l'égalité politique susceptible d'être protégée par l'article 15.

L'article 24 de la loi fédérale stipule que tout parti politique doit être enregistré et doit présenter cinquante candidats dans les délais prescrits; mais l'article 28 ajoute que le directeur général des élections peut lors d'une élection générale, radier du registre le parti qui n'a pas été représenté depuis la dernière élection par au moins douze députés.

On peut se demander si ces conditions ne sont pas trop restrictives et ne tendent pas trop à l'élimination des partis minoritaires. L'objectif du législateur est certes de valoriser l'expression électorale en ne retenant que les partis ayant suffisamment de consistance pour bénéficier des

61. Id. à la p. 267. 
avantages que procure la loi aux partis reconnus; la loi décourage l'émergence de groupuscules. Le multipartisme est certes favorisé par l'idéal démocratique que véhicule la Charte, mais non pas l'instabilité politique qui résulte souvent de la multiplication des partis, d'ailleurs étrangère à notre évolution politique. Le nombre de cinquante candidats est-il déraisonnablement élevé? Il favorise, certes, l'émergence de partis d'envergure nationale, ce qui est normal dans un État fédéral. Peut-on en dire autant du parti qui ne réussit pas à faire élire douze députés? La question est assez épineuse car les avantages attachés au statut de parti enregistré sont considérables. Certes, le fait de ne pas être regroupé en partis reconnus n'empêche pas les candidats de toutes tendances politiques de briguer les suffrages et de bénéficier des avantages destinés à tout autre candidat. Il s'agit de candidats indépendants qui ont droit au remboursement de dépenses électorales, par exemple. Ils sont certes privés du support d'un parti reconnu, mais leurs droits politiques fondamentaux sont intacts.

Au Québec, la loi électorale prévoit qu'un parti peut voir son autorisation retirée par le directeur général des élections s'il ne présente pas dix candidats aux élections générales ou s'il ne se conforme pas aux exigences de la loi en matière de renseignements requis aux fins de mise à jour des registres de vérification ou d'acquittements de ses dépenses, etc. Ces dispositions de la loi ont été contestées en Cour supérieure parce qu'enfreignant la liberté d'expression protégée par l'article $2 b$ ) de la Charte. La Cour admet qu'il y a là restriction à une forme de liberté d'expression «au nom de la protection du public en matière de financement des partis politiques ${ }^{62}$. Néanmoins, cette restriction est raisonnable et se justifie dans une société libre et démocratique:

«L'intégrité du processus électoral constitue une condition essentielle au maintien de notre société libre et démocratique. L'État a donc le devoir de veiller à protéger l'intégrité et la crédibilité de ses mécanismes électoraux. Les dispositions relatives au financement des partis politiques et à l'autorisation des partis politiques traduisent cette double préoccupation» ${ }^{63}$.

La situation faite aux partis non reconnus est-elle discriminatoire au regard de l'article 15 de la Charte? En Ontario, où la loi électorale est

62. Parti Union Nationale c. Côté, [1989] R.J.Q., 2502, 2512 (j. Rousseau-Houle) (C.S.
63. Qué). 
assez analogue à la loi fédérale, il y avait, en 1985, huit partis reconnus et vingt-sept non reconnus. Toutefois selon la loi ontarienne, un parti en émergence peut se faire reconnaître s'il produit une liste de dix mille signatures d'électeurs ${ }^{64}$. La distinction que la loi fait entre partis reconnus et non reconnus est-elle discriminatoire, en ce sens qu'elle serait fondée sur des considérations politiques? Nous ne le croyons pas. Les exigences sont les mêmes pour tous les partis sans égard à leurs opinions, leurs programmes ou leurs doctrines. C'est plutôt au plan de la liberté d'expression en matière politique qu'il faut évaluer les interdictions faites aux partis non reconnus ainsi que les avantages qui leurs sont réservés: c'est sous cet angle que nous en traiterons.

\subsection{LE PLAFONNEMENT ET LE CONTRÔLE DES DÉPENSES ÉLECTORALES}

La loi électorale impose diverses restrictions aux dépenses électorales. La définition même du concept, la réglementation de la façon selon laquelle ces dépenses doivent être engagées, la vérification et le contrôle exigés par la loi restreignent à certains égards la liberté politique. Mais ce qui est le plus controversé c'est le plafonnement de ces dépenses effectuées soit par les candidats soit par les partis. Aux États-Unis, en 1976 la Cour suprême a jugé que ce plafonnement violait la liberté d'expression garantie par le Bill of Rights ${ }^{65}$.

Cette question a tout d'abord été abordée au Québec en regard de l'article 3 de la Charte québécoise qui est quasi identique à l'article 2 de la Charte canadienne. La Cour supérieure ${ }^{66}$, puis la Cour d'appel ${ }^{67}$ ont considéré que la liberté d'expression ne comprend pas la liberté de faire des dépenses à sa guise pour s'exprimer.

Cette conception restrictive semble difficilement compatible avec l'interprétation libérale qu'a donnée la Cour suprême depuis 1984 à la notion «d'expression» dans plusieurs de ses arrêts.

47. Keith D. Ewing «The Funding of Political Parties in Ontario», (1989) 27 Osgoode Hall

L.J. 27,35.

65. Buckley c. Valeo, 424 U.S.1, 96 S.Ct. 612 (1976).

66. Roberge c. Québec (P.G.), C.S. Montréal, no 500-05-0004628-804 16 avril 1980; Boucher c. C.E.Q., [1982] C.S.P. 1003.

67. Roberge c. Québec (P.G.), C.A. 18 nov. 1987, C.A.M. 09-000175-828. 
La Cour Banc de la Reine de l'Alberta n'eut pas de difficulté, en 1984, à conclure que toute limitation aux dépenses électorales concerne la liberté d'expression ${ }^{68}$. On contestait devant elle la validité des dispositions de la loi électorale fédérale qui interdisent à toute autre personne que les candidats et partis politiques de faire des dépenses électorales au sens de la loi. L'argumentation du Gouvernement était que le législateur, se fondant sur le Comité Barbeau et le Comité Chappel, avait afin d'améliorer le système électoral, limité les dépenses électorales des candidats et des partis. Cet objectif serait complètement détruit si les tiers pouvaient engager de telles dépenses de façon illimitée. La Cour se contenta de répondre qu'il ne lui appartenait pas de considérer s'il n'y avait pas d'autres moyens d'atteindre le même objectif et qu'il ne lui appartenait pas de réécrire la législation pour la rendre conforme à la Charte. Elle annula les articles 70 et 72 et la loi.

Cette décision est, à notre avis, d'une très grande faiblesse. Certes le juge n'avait pas le bénéfice de l'arrêt Oakes. Car si l'on applique aujourd'hui le test de l'arrêt Oakes, on peut difficilement arriver à une conclusion semblable. La disposition contestée est non seulement rationnelle, mais c'est la seule qui soit appropriée à l'atteinte de l'objectif du législateur par le moyen principal envisagé, soit le plafonnement des dépenses électorales. Or dans cette affaire, ce n'était pas le plafonnement qui était contesté, mais le fait que seuls les partis et les candidats puissent faire des dépenses électorales.

La loi québécoise, comme d'ailleurs les autres lois électorales, prévoit un régime de contrôle et de vérification des dépenses des partis et candidats. La Cour supérieure du Québec a décrété que ces dispositions restreignent, certes, la liberté d'expression politique mais sont justifiées par la nécessité pour l'État de maintenir «l'intégrité du processus électoral» ${ }^{69}$.

\subsection{LE FINANCEMENT DES CANDIDATS ET DES PARTIS}

Comme il est admis depuis longtemps que les élections ne se font pas sans argent qui est, là comme ailleurs, le nerf de la guerre, deux questions se sont soulevées ces dernières décennies: la question du financement public des candidats et partis ainsi que celle du contrôle des

68. National Citizen's Coalition Inc. c. Canada (P.G.) (1984), 11 D.L.R. (4th) 481.

69. Parti Union Nationale c. Côté, supra, note 58. 
contributions provenant des particuliers, des entreprises, des syndicats ou du grand public.

\subsubsection{LE FINANCEMENT GOUVERNEMENTAL}

Cette question a été étudiée par la Cour suprême des États-Unis en 1976, dans Buckley c. Valeo ${ }^{70}$. La Cour a conclu à la validité constitutionnelle de la disposition prévoyant le financement de certaines dépenses électorales à même les fonds publics, la qualifiant ainsi:

«a congressional effort, not to abridge, restrict, or censor speech, but rather to use public money to facilitate and enlarge public discussion and participation in the electoral process, goals vital to a self-governing people» ${ }^{71}$.

La loi électorale du Manitoba contenant une telle disposition a été contestée en 1985. La Cour du Banc de la Reine, puis la Cour d'appel de la province ont rejeté le pourvoi. ${ }^{72}$ La Cour d'appel s'est majoritairement appuyée sur la jurisprudence américaine. Elle estima que le fait pour l'État d'affecter ainsi des fonds publics, n'équivalait pas à requérir des citoyens des contributions obligatoires destinées à telle ou telle fin spécifique: le gouvernement a la responsabilité d'affecter des fonds à d'innombrables programmes qui plaisent ou déplaisent. D'autre part, le fait que les fonds publics supportent l'expression d'opinions minoritaires n'a pas pour effet d'inciter la majorité à adhérer à ces opinions: chacun reste libre d'y adhérer ou non. En un mot, la liberté d'opinion garantie par la Charte n'est pas affectée par le fait que l'État accorde une aide financière raisonnable à l'expression de tendances politiques variées.

La Cour suprême du Canada a subséquemment rejeté le pourvoi mais sans se prononcer vraiment sur la question ${ }^{73}$. Elle a estimé qu'aucun élément de preuve n'a été présenté à l'appui de cette plainte. L'appelant n'a pas établi les conséquences préjudiciables découlant de la prétendue violation de la Charte par la législature: il n'avance que des hypothèses ou conjectures.

\footnotetext{
70. Supra, note 61.

71. Id. à la p. 670.

72. Re MacKay and Manitoba (1985), 19 D.L.R. (4th) 185 (Man. Q.B.), (1985) 24 D.L.R.(4th) 587 (Man. C.A.).

73. MacKay c. Manitoba (P.G.), [1989] 2 R.C.S. 357.
} 
À notre avis, il est difficile de qualifier ce genre de législation comme constituant une atteinte à la liberté d'opinion. Il s'agit d'une mesure poursuivant un objectif valable et légitime mais dont l'effet est neutre en ce qui concerne l'État: celui-ci n'impose aucun dirigisme et n'exclut pas a priori quelque tendance politique que ce soit.

Enfin, il faut tenir compte qu'une telle mesure législative s'inscrit dans un ensemble cohérent qui comprend l'enregistrement des partis politiques, la réglementation et le plafonnement des dépenses électorales et, dans certaines provinces, des restrictions aux contributions politiques.

Au Québec, la loi de $1989^{74}$ reconduit un système créé il y a une quinzaine d'années sous le nom de «financement public des partis politiques». Il s'agit d'une allocation aux partis politiques autorisés, déterminée par le directeur général des élections, qui «vise à rembourser les partis des frais engagés pour leur administration courante, pour la diffusion de leur programme politique et pour la coordination de l'action politique de leurs membres $\gg{ }^{75}$. Ces partis doivent être autorisés et se qualifier en termes de pourcentage des votes obtenus. Ces contributions sont limitées et l'allocation n'est acceptable que pour les frais réellement engagés.

\subsubsection{LES CONTRIBUTIONS POLITIQUES}

L'idée de limiter et de contrôler, à certains égards, les contributions politiques et électorales est venue de la volonté du législateur d'assainir les moeurs politiques et électorales et de valoriser l'expression politique de façon plus égalitaire.

La Loi électorale québécoise ${ }^{76}$ de 1989 contient toute une réglementation des procédés de contributions à des fins politiques en faveur des partis et des candidats. Deux idées dominent cette réglementation: premièrement, seul un électeur peut contribuer et à même ses propres biens; deuxièmement, les contributions sont plafonnées à \$3000 par année civile en faveur de chaque parti et candidat indépendant.

La première idée vise à interdire les contributions corporatives et syndicales et à favoriser le financement populaire des partis et candidats;

\footnotetext{
74. L.R.Q. 1989 , c. 1 , art. 81 à 86.

75. Id., art. 83.

76. L.Q. 1989, c. 1, art. 87 à 101.
} 
cela implique, à certains égards, une certaine divulgation de la provenance des contributions.

La Cour suprême des États-Unis s'est prononcée sur une question similaire dans l'arrêt Bellotti ${ }^{77}$ par une majorité de 5 contre 4 . Il s'agissait d'une loi du Massachusetts qui interdisait toute contribution ou dépense corporative lors d'un référendum, à moins que le sujet intéresse particulièrement la corporation contributrice. Le gouvernement prétendait que la participation des corporations exerçait une influence indue sur l'issue du référendum et, à la fin, détruirait la confiance du peuple dans le processus démocratique et l'intégrité du gouvernement. La Cour répondit ceci:

«[As we noted in Buckley] the concept that government may restrict the speech of some elements of our society in order to enhance the relative voice of others is wholly foreign to the First Amendement (...) the people in our democracy are entrusted with the responsibility for judging and evaluating the relative merits of conflicting arguments. They may consider in making their judgment, the source and credibility of the advocate. But if there be any danger that the people cannot evaluate the information and arguments advanced by [corporations], it is a danger contemplated by the Framers of the First Amendement ${ }^{78}$.

La Cour ajoute toutefois que le gouvernement pouvait démontrer que la participation des corporations «threatened imminently to undermine democratic processes, thereby denigrating rather than serving First Amendment interests, these arguments would merit our consideration ${ }^{79}$.

Tout comme la Cour suprême canadienne dans Mackay ${ }^{80}$, la Cour américaine n'exclut pas la possibilité d'une contestation de ce genre de législation à condition que l'on présente un dossier étoffé. Ici, il appartient au gouvernement de démontrer que les contributions corporatives faussent vraiment le processus démocratique et constituent une menace imminente à l'intégrité de ce processus.

77. First National Bank of Boston c. Bellotti, 435 U.S. 765, 98 S.Ct. 1407 (1978)

78. Id. aux pp. 1423-1424.

79. Id. à la p. 1423 .

80. Supra, note 69 . 
Si l'on applique le test de l'arrêt Oakes, le gouvernement doit démontrer que l'interdiction absolue des contributions corporatives est de nature à porter le moins possible atteinte au droit en question, et que les effets de cette mesure sont proportionnels à l'objectif poursuivi. Or suivant l'exigence posée par la Cour, le gouvernement arrivera difficilement à convaincre la Cour qu'il ne peut arriver aux même fins par des moyens moins draconiens. Elle raisonnera vraisemblablement comme elle l'a fait dans l'arrêt Ford $^{81}$. La réponse implicite qui se dégage c'est que le législateur pourrait tenter de circonscrire, de plafonner, de réglementer les contributions corporatives plutôt que de les interdire complètement. L'Ontario a, à cet égard, une législation qui nous paraît respectueuse de la Charte: la loi n'interdit pas les contributions corporatives et syndicales mais les plafonne et prévoit des normes destinées à prévenir les évasions ${ }^{82}$.

La question de savoir si une corporation peut faire des contributions politiques sans l'assentiment de ses actionnaires ou contre leurs convictions, de même que celle de savoir si un syndicat accrédité peut distraire, en faveur des partis politiques, les fonds syndicaux recueillis par retenue obligatoire suivant la formule Rand, ne sont pas régies directement par la législation électorale; aussi nous ne l'étudierons pas expressément. Cette question concerne la liberté politique des individus face au pouvoir corporatif ou syndical ${ }^{83}$.

\subsection{LA PUBLICITÉ ÉLECTORALE}

Trois dispositions de la loi fédérale actuelle traitent de la publicité électorale. L'article 157 interdit d'utiliser, le jour du scrutin, un système de sonorisation ou de haut-parleurs à des fins électorales sur un véhicule ou à portée de voix d'un bureau de scrutin. L'article 158 interdit d'afficher ou d'exhiber en tout endroit à l'intérieur ou à l'extérieur du bureau de scrutin tout matériel électoral, enseigne, étiquette, affiche, etc. Enfin, en vertu de l'article 159, le scrutateur peut expulser d'un bureau de scrutin toute personne qui enfreint ces dispositions et faire enlever du bureau tout

\footnotetext{
81. Supra, note 18.

82. $\quad$ Supra note 4, p. 37 ss.

83. Dans le cas des syndicats, la question a été étudiée dans Re Lavigne and Ont. Public Service Employee Union (1986), 29 D.L.R. (4th) 321, (1989) 67 O.R. (2d) 536 (Ont. C.A.). En droit du travail canadien, la retenue obligatoire des cotisations syndicales chez tous les travailleurs et le monopole syndical ont été jusqu'ici jugés compatibles avec la Charte.
} 
objet utilisé en dérogation de ces dispositions. Des sanctions pénales sont prévues en cas de violation.

Ces dispositions limitent le droit à la liberté d'expression de l'article 2 de la Charte, mais l'objectif poursuivi peut facilement être considéré comme important: il s'agit de favoriser l'expression électorale sans la moindre pression. S'agit-il d'une mesure trop radicale et disproportionnée par ses effets, par rapport à l'objectif poursuivi? Certes, la mesure est radicale, mais l'objectif est tellement important que tout doit être mis en oeuvre pour assurer en toute quiétude la démarche et le choix de l'électeur. D'autre part, les candidats et les partis ont eu toute la campagne électorale pour utiliser ces moyens de propagande. De plus, le jour même du scrutin ils peuvent continuer par des contacts personnels leur travail de persuasion de l'électeur.

À notre avis, le gouvernement peut facilement démontrer que les articles 157 et 158 de la loi se justifient dans le cadre d'une société libre et démocratique. De toute façon, l'interdiction de toute publicité électorale le jour du scrutin est profondément inscrite dans nos moeurs politiques. Il importe de mentionner que par publicité électorale, l'article 158 n'englobe pas la publication dans la presse ou la diffusion dans les médias de messages comportant des opinions politiques ou un appui à un parti ou un candidat, ou aux opinions politiques de ces derniers.

Il faut faire une nette distinction entre la publicité et le discours politique ordinaire, couvert par la liberté de reportage ou la liberté éditoriale, suivant les termes utilisés par la Haute Cour de justice de l'Ontario dans une décision de $1988^{84}$. La liberté d'expression en matière politique a été consacrée par la jurisprudence constitutionnelle bien avant la Charte de $1982^{85}$. Depuis 1982, la Cour suprême a eu à se prononcer sur la liberté du discours commercial ${ }^{86}$ et sur la liberté de la presse dans divers domaines, notamment le domaine judiciaire ${ }^{87}$. Si une certaine réglementation de la publicité électorale s'impose et se justifie dans le

84. Trieger c. Canadian Broadcasting Corp. (1988), 66 O.R. (2d) 273, 278 (j. Campbell). 85. Re Alberta Statutes, [1938] R.C.S. 100.

86. Voir Clare F. Beckton, «Liberté d'expression» dans Gérald A. Beaudoin et Edward Ratushny (dir.), op. cit., note 1, pp. 225-259; aussi Neil Finkelstein et Brian McLeod Rogers (dir.), Charter Issues in civil cases, Toronto, Carswell 1988; voir notamment l'arrêt Ford c. Québec (P.G.), supra, note 18.

87. Allen M. Linden et Philip Anisman (dir.), The Media, The Courts and The Charter, Toronto, Carswell 1986; voir Edmonton Journal c. Alberta (P.G.), [1989] 2 R.C.S. 1326; Canadian Newspapers Co. c. Canada (P.G.), [1988] 2 R.C.S 122. 
cadre d'une société libre et démocratique, il en va tout autrement du discours politique ordinaire.

\subsection{LA COMMUNICATION AUDIO-VISUELLE}

La loi électorale fédérale contient une vingtaine d'articles sur «les émissions politiques» ou «political broadcasts» c'est-à-dire la radiodiffusion et télédiffusion d'émissions politiques en période électorale (articles 303 à 322). Ces dispositions renvoient toutefois à la Loi sur la radiodiffusion et à ses règlements d'application ${ }^{88}$. La Loi sur la radiodiffusion déclare que le système canadien doit sauvegarder, enrichir et renforcer la structure politique du Canada, favoriser le droit à la liberté d'expression d'opinions divergentes sur des sujets d'intérêt public, etc. La loi confère au Conseil de la radiodiffusion et des télécommunications canadiennes le pouvoir de réglementer les normes des émissions et l'attribution du temps d'antenne pour donner effet à ces objectifs. Le Conseil peut également par règlement régir la nature de la publicité et le temps qui peut y être consacré, fixer la proportion du temps d'antenne pouvant être consacrée à la radiodiffusion de messages publicitaires, d'annonces politiques ou d'émissions de nature partisane, ainsi que déterminer la répartition équitable de ce temps entre les partis politiques et les candidats. Le règlement de 1987 sur la télédiffusion ${ }^{89}$ ajoute qu'«au cours d'une période électorale, un diffuseur doit répartir équitablement entre les différents partis politiques accrédités et les candidats rivaux (...) le temps consacré à la radiodiffusion d'émissions, d'annonces ou d'avis qui exposent la politique d'un parti». Enfin, le C.R.T.C. a émis le 2 septembre 1988 un énoncé de politique dans lequel il précise que l'équité s'applique à la durée des émissions, à l'horaire, à l'auditoire possible, à la langue, à la couverture des questions et à leur traitement, ainsi qu'aux prix $^{90}$.

Ces deux derniers textes ont été contestés au cours de la campagne électorale de novembre 1988 par le chef du Parti Vert qui n'avait pas été invité à participer à un débat des chefs des trois grands partis canadiens. La Haute Cour de l'Ontario rejeta la demande d'injonction, logée d'ailleurs l'avant-veille du débat télévisé, pour deux

\footnotetext{
88. S.R.C. 1985 , c. B-9.

89. (1987) 121 Gaz. Can. II, 343, DORS 87-49, art. 8; voir au même effet le règlement sur la radiodiffusion de 1987.

90. C.R.T.C., Avis public n. 88-142 et Circulaire n. 334.
} 
raisons ${ }^{91}$. Premièrement, la plainte devrait relever en premier lieu du C.R.T.C., chargé par la loi de voir si les télédiffuseurs ont agi avec équité. Deuxièmement, la Charte, invoquée par le requérant, garantit le droit à la liberté d'expression mais non celui de requérir la propriété d'autrui pour véhiculer ses opinions. Se fondant sur la jurisprudence de la Cour fédérale ${ }^{92}$, ainsi que sur un arrêt de la Cour suprême des États-Unis ${ }^{93}$, le juge estime que la Constitution ne confert pas un droit d'accès aux médias. Enfin, comme le débat télévisé résulte d'un accord négocié entre certaines grandes chaînes et les trois grands partis, une intervention judiciaire en l'espèce serait une interférence directe dans le débat politique, notamment en dictant aux télédiffuseurs ce qu'il est opportun de diffuser, et en imposant aux chefs politiques de débattre avec des personnes qu'ils n'auraient pas choisies.

La Cour n'a pas fermé complètement la porte à une contestation appropriée, mais sur la base d'un dossier mieux étoffé. Elle a cependant rejeté sommairement un autre argument fondé sur l'article 15 de la Charte, soit le droit à l'égalité.

Le règlement du C.R.T.C. se borne à imposer la règle de la répartition équitable en faveur des partis accrédités et des candidats. $\mathrm{La}$ Loi électorale, pour sa part, oblige en période électorale, les radiodiffuseurs à libérer pour achat un total de six heures et demi de temps d'émission, aux heures de grande écoute, en faveur des partis enregistrés. Un système d'arbitrage est prévu. La loi prévoit aussi du temps d'antenne gratuit. Les partis enregistrés sont seuls bénéficiaires de ces avantages. Il s'agit là d'une question qui devra faire l'objet de reconsidération en regard de l'ensemble de la jurisprudence de la Cour suprême sur l'article 15 .

\section{CONCLUSION}

Une campagne électorale nationale au Canada c'est une vaste opération qui dure 50 jours, met en présence quelque 1600 candidats dans 295 circonscriptions, sur un immense territoire, implique 110000 recenseurs, un personnel électoral considérable et un budget public de $\$ 30$ millions. Parallèlement, dans chacune des dix provinces et dans les deux territoires fédéraux, une opération analogue se déclenche tous les

91. Supra, note 80.

92. Re: New Brunswick Broadcasting Co. c. C.R.T.C., [1984] 2 C.F. 410 (C.F.A.).

93. Colombia Broadcasting System Inc. c. F.C.C., 453 U.S. 367 (1981). 
quatres ans, sinon plus souvent. Les lois électorales qui encadrent ces vastes opérations ont été modernisées et ajustées aux éxigences contemporaines, mais la Charte constitutionnelle de 1982 soulève d'autres questions qui n'ont pas encore été complètement élucidées, même si la reflexion progresse.

La question de l'octroi du droit de vote aux prisonniers, aux juges et aux handicapés mentaux ne devrait pas tellement soulever de difficultés en vue d'une réforme imminente de la Loi électorale. À notre avis, la suppression du droit de vote chez tous les prisonniers de même que chez les criminels est contraire à la Charte.

Comme l'a soutenu le juge Marshall de la Cour suprême des États-Unis, retirer le droit de vote à ceux qui sont pratiquement hostiles à l'ordre établi afin qu'ils n'aient aucune influence politique «strikes at the very heart of the democratic process ${ }^{94}$.

Une seule exception nous paraît légitime, celle des personnes condamnées pour manoeuvres électorales frauduleuses. La loi devrait identifier expressément ces infractions ou permettre au juge de les qualifier ainsi; la perte du droit de vote pour une période déterminée devrait être encourue par l'effet de la loi. D'ailleurs, cette condamnation devrait faire encourir la perte du droit de se porter candidat et de se livrer à un travail partisan pendant la même période.

Quant à l'ensemble de la population carcérale, la loi doit prévoir l'aménagement de l'exercice du droit de vote ainsi que les situations exceptionnelles où cela ne serait pas possible. Pour ce qui est du cas des handicapés mentaux, la loi devrait retenir le critère de la capacité effective pour l'électeur d'exercer par lui-même un choix sans assistance. Les handicapés mentaux de même que les personnes confuses pour raison d'âge ou autre conservent leur droit de vote mais c'est à eux de l'exercer personnellement par une demande lucide. La situation est essentiellement différente des handicapés physiques qui peuvent avoir besoin d'assistance: le scrutateur ou l'agent qui assiste l'électeur ne fait qu'exécuter l'ordre donné par ce dernier.

94. Richardson c. Ramirez, 418 U.S. 24, 94 S.Ct. 2655, 2685 (1974) : le juge Marshall était cependant dissident dans cette affaire. 
Quant aux conditions d'exercice de suffrage, telle la résidence, l'inscription sur les listes électorales, l'absence temporaire du lieu de résidence, le vote par anticipation, nos législations de même que le projet de loi C-79 nous paraissent comporter des normes acceptables au regard de la Charte. Le vote des absents n'existe au fédéral que dans le cas des militaires et du personnel diplomatique; le Québec vient de l'étendre en 1989 à l'ensemble des québécois vivant ou en déplacement à l'étranger, mais à certaines conditions. Il nous paraît évident que la Charte n'impose pas au législateur de permettre aux Canadiens en déplacement aux quatres coins de la planète d'exercer leur droit de vote. On s'est apitoyé sur le sort des quelques 500000 Canadiens qui vont l'hiver se faire dorer sous le soleil de la Floride ou des Antilles... Si le législateur veut permettre dans des conditions raisonnables et efficaces l'exercice du suffrage par les absents, cela l'honore, mais il n'appartient pas au juge constitutionel de l'imposer.

Le droit d'éligibilité et de candidature est consacré par la Charte mais il est normal que les juges et les députés provinciaux ne puissent briguer les suffrages. La situation des fonctionnaires est différente. Nous avons conclu que l'octroi d'un congé électoral s'impose pour l'ensemble des fonctionnaires, sous réserve de certains aménagements quant à la réintégration dans la fonction publique au terme d'un tel congé.

Pour valoriser l'expression électorale, les législateurs contemporains ont imaginé un régime juridique des partis politiques: le régime fédéral actuel nous paraît assez conforme à la Charte. Les législateurs ont aussi imposé un plafonnement des dépenses électorales. À notre avis, la loi actuelle comporte des mesures rationnelles et proportionnées à l'atteinte des objectifs légitimes du législateur.

Le financement public de certaines dépenses électorales des partis et candidats ne constitue pas, à notre avis, une atteinte à la liberté d'opinion. Cette mesure s'inscrit dans un ensemble cohérent qui comprend l'enregistrement des partis, la réglementation et le plafonnement des dépenses électorales. L'objectif du législateur est valable et la mesure a un effet neutre en ce qui concerne l'État; celle-ci n'implique aucun dirigisme et n'exclut a priori aucune tendance politique.

Le contrôle des contributions politiques, notamment l'interdiction des contributions émanant des corporations ou des syndicats, soulève une difficulté au regard de la liberté d'expression consacrée par la Charte. À notre avis, l'interdiction absolue a de fortes chances d'être jugée contraire 
(1991) 21 R.D.U.S.

à la Charte, parce que non proportionnelle à l'objectif poursuivi par le législateur. Si l'on se réfère à l'esprit de la jurisprudence récente de la Cour suprême, l'interdiction absolue pourrait être jugée trop radicale. Cette question devrait faire l'objet d'études plus approfondies. 